\title{
A NOTE ON COUNTABLY GENERATED COMPLETE BOOLEAN ALGEBRAS
}

\author{
THOMAS J. JECH
}

\begin{abstract}
In this note, we construct (assuming the G.C.H.) a countably generated cardinal preserving complete Boolean algebra of cardinality $\kappa_{\omega+2}$.
\end{abstract}

1. The problem. Throughout the paper we assume that the reader is familiar with basic properties of complete Boolean algebras and with basic facts about the method of forcing and Boolean valued models (see e.g. [3]).

A complete Boolean algebra (cBa) is countably generated, if there exists a countable set $X \subset B$ (of generators) such that $B$ is the least complete subalgebra of $B$ that includes $X$.

A theorem of Gaifman-Hales [1], [2] shows that for any cardinal number $\kappa$, there exists a countably generated $\mathrm{cBa} B$ of cardinality $|B| \geqslant \kappa$. In fact, every $\mathrm{cBa}$ can be embedded in a countably generated $\mathrm{cBa}$ (Kripke [5]).

The algebra constructed in the proof of the Gaifman-Hales Theorem, and all subsequent related constructions [6], [5], [4], have the property that the corresponding Boolean valued model collapses cardinals; one constructs a $\mathrm{cBa} B$ of size $\kappa$ and the ordinals below $\kappa$ (or even $\kappa$ itself) become countable in $V^{B}$.

Thus it is natural that we address ourselves to the question whether it is possible to construct a countably generated $\mathrm{cBa}$ of arbitrarily large cardinality without collapsing cardinals in $V^{B}$.

Definition. A complete Boolean algebra $B$ is cardinal preserving if for every cardinal number $\kappa, \llbracket \check{\kappa}$ is a cardinal $\rrbracket_{B}=1$.

Below, we give a construction of a countably generated cardinal preserving $\mathrm{cBa}$ of cardinality $\boldsymbol{N}_{\omega+2}$ (the largest cardinality I can do). The method is a modification of Solovay's almost disjoint forcing. The general problem, existence of arbitrarily large c.g.c.p.c.B.a. remains open.

2. The theorem. Assume the generalized continuum hypothesis. There is a countably generated cardinal preserving complete Boolean algebra $B$ such that $|B|=\kappa_{\omega+2}$.

3. The proof. The method is basically the almost disjoint forcing of Solovay. Thus from now on we assume familiarity with the construction [4, $\S 2$ ]. We construct a set of forcing conditions $P$ such that the associated $\mathrm{cBa}$ $B=$ r.o. $P$ is (a) countably generated, (b) cardinal preserving, and (c) of cardinality $\boldsymbol{N}_{\omega+2}$.

Received by the editors March 10, 1975.

AMS (MOS) subject classifications (1970). Primary 02K99; Secondary 06A40.

๑ American Mathematical Society 1976 
To begin with, we review Solovay's method of coding a subset of $\omega_{1}$ by a generic subset of $\omega$.

Let $\Re \subseteq \Re$ be models of ZFC such that $\omega_{1}^{\Re}=\omega_{1}^{\Re}$ and let $A$ be a subset of this $\omega_{1}, A \in \Re$ (possibly $A \notin \Re$ ). Then there is $x \subseteq \omega$, generic over $\Re$, such that $A \in \Re[x]$.

The generic set $x$ is obtained as follows: Let $Y=\left\{y_{\alpha}: \alpha<\omega_{1}\right\}$ be a family of almost disjoint infinite subsets of $\omega, Y \in \Re$. (Almost disjoint means that $y_{\alpha} \cap y_{\beta}$ is finite whenever $\alpha \neq \beta$.) The forcing conditions are chosen as to produce a set $x \subset \omega$ such that

$$
A=\left\{\alpha<\omega_{1}: x \cap y_{\alpha} \text { is finite }\right\} .
$$

The set of forcing conditions $P_{A}$ is defined as follows:

$p \in P_{A}$ iff $p=(f, t)$ where

(i) $f$ is a 0 -1-function whose domain is a finite subset of $\omega$

(ii) $t$ is a finite subset of $A$.

$$
(f, t) \leqslant\left(f^{\prime}, t^{\prime}\right) \text { iff }
$$

(i) $f^{\prime} \subseteq f$,

$$
\text { (ii) } t^{\prime} \subseteq t \text {; }
$$$$
\text { if } s=\{i: f(i)=1\} \text { and } s^{\prime}=\left\{i: f^{\prime}(i)=1\right\} \text { then }
$$$$
\text { (iii) } s^{\prime} \cap y_{\alpha}=s \cap y_{\alpha} \text { for all } \alpha \in t \text {. }
$$

If $G$ is an $\Re$-generic subset of $P_{A}$, and

$$
x=\{i<\omega: \exists(f, t) \in G \text { such that } f(i)=1\},
$$

then

$$
\begin{array}{r}
G=\{(f, t): \text { if } s=\{i: f(i)=1\}, \text { then } s \text { is an initial segment of } x, \\
\text { and } \left.x \cap y_{\alpha}=s \cap y_{\alpha} \text { for all } \alpha \in t\right\},
\end{array}
$$

and we have $\Re[x]=\Re[G]$. An elementary forcing argument shows that $x$ satisfies (3.1) and the models $\Re$ and $\varkappa[x]$ have the same cardinals (see [4]). The latter assertion holds in view of $\left(P_{A}, \leqslant\right)$ satisfying the countable chain condition.

The above construction can obviously be generalized to higher cardinals, in particular when $\kappa_{0}$ is replaced by $\kappa_{n}$.

Let $n>0$ and let $\Re \subseteq \Re$ be models with same cardinals $\leqslant \aleph_{n+1}$, assume that $\Re=2^{\kappa_{n-1}}=\kappa_{n}$ and let $A$ be a subset of $\omega_{n+1}$ (i.e. $\omega_{n+1}^{\Re}$ ). Let $\left\langle y_{\alpha}^{(n)}: \alpha<\omega_{n+1}\right\rangle \in \mathfrak{N}$ be a family of subsets of $\omega_{n}$ such that $\left|y_{\alpha}^{(n)}\right|=\aleph_{n}$ for each $\alpha$, and $\left|y_{\alpha}^{(n)} \cap y_{\beta}^{(n)}\right|<\kappa_{n}$ whenever $\alpha \neq \beta$. (It is well known that a family of $\kappa_{n+1}$ "almost disjoint" subsets of $\omega_{n}$ exists if $2^{\boldsymbol{\kappa}_{n-1}}=\kappa_{n}$.) Given $A \subseteq \omega_{n+1}, A \in \mathcal{N}$, let $P_{A}^{(n)}$ be the following set of forcing conditions:

$$
p^{(n)} \in P_{A}^{(n)} \text { iff } p^{(n)}=\left(f^{(n)}, t^{(n)}\right) \text { where }
$$

$$
\begin{aligned}
& \text { (i) } f^{(n)} \text { is a } 0 \text {-1-function on a subset of } \omega_{n} \text {, and }\left|\operatorname{dom} f^{(n)}\right|<\aleph_{n} \text {, } \\
& \text { (ii) } t^{(n)} \subset A,\left|t^{(n)}\right|<\aleph_{n} \text {. }
\end{aligned}
$$




$$
\left(f^{(n)}, t^{(n)}\right) \leqslant\left(f_{1}^{(n)}, t_{1}^{(n)}\right) \text { if }
$$

(i) $f_{1}^{(n)} \subseteq f^{(n)}$,

(ii) $t_{1}^{(n)} \subseteq t^{(n)}$,

(iii) if $s^{(n)}=\left\{i: f^{(n)}(i)=1\right\}$ and $s_{1}^{(n)}=\left\{i: f_{1}^{(n)}(i)=1\right\}$, then $s_{1}^{(n)} \cap y_{\alpha}^{(n)}=s^{(n)} \cap y_{\alpha}^{(n)}$ for all $\alpha \in t^{(n)}$.

If $G_{n}$ is an $\Re$-generic subset of $P_{A}^{(n)}$, and

$$
x_{n}=\left\{i<\omega_{n}: \exists\left(f^{(n)}, t^{(n)}\right) \in G_{n} \text { such that } f^{(n)}(i)=1\right\},
$$

then $\mathscr{N}\left[x_{n}\right]=\mathscr{U}\left[G_{n}\right]$. The same argument as in [4] shows that

$$
A=\left\{\alpha<\omega_{n+1}:\left|x_{n} \cap y_{\alpha}^{(n)}\right|<\kappa_{n}\right\} \text {. }
$$

Since $2^{\kappa_{n-1}}=\kappa_{n}$ in $\Re, P_{A}^{(n)}$ satisfies the $\kappa_{n+1}$-chain condition; moreover, every descending sequence of conditions of length $\left\langle\kappa_{n}\right.$ has a lower bound and so $\Re[x]$ and $\Re$ have same subsets of $x_{n-1}$. Thus $\Re$ and $\Re\left[x_{n}\right]$ have the same cardinals.

We are now ready to construct a set of forcing conditions $P$ such that $B=$ r.o. $P$ satisfies (a), (b) and (c).

Let us fix, for every $n$, a family

$$
\left\langle y_{\alpha}^{(n)}: \alpha<\omega_{n+1}\right\rangle
$$

of subsets of $\omega_{n}$ such that $\left|y_{\alpha}^{(n)} \cap y_{\beta}^{(n)}\right|<\kappa_{n}$ iff $\alpha \neq \beta$.

The set $P$ consists of all sequences

$$
p=\left\langle p^{(n)}: n\langle\omega\rangle\right.
$$

such that each $p^{(n)}=\left(f^{(n)}, t^{(n)}\right)$ where

(i) $f^{(n)}$ is a 0-1-function on a subset of $\omega_{n}$ and $\left|\operatorname{dom} f^{(n)}\right|<\aleph_{n}$,

$$
\begin{aligned}
& \text { (ii) } t^{(n)} \subset \omega_{n+1},\left|t^{(n)}\right|<\kappa_{n}, \\
& \text { (iii) } t^{(n)} \subseteq s^{(n+1)} \text { where } s^{(n+1)}=\left\{i<\omega_{n+1}: f^{(n+1)}(i)=1\right\} .
\end{aligned}
$$

A condition $p=\left\langle p^{(n)}: n\langle\omega\rangle\right.$ is stronger than $q=\left\langle q^{(n)}: n\langle\omega\rangle, p \leqslant q\right.$, if $p^{(n)} \leqslant q^{(n)}$ in $P^{(n)}$, for every $n$ (see (3.6)).

Let $G$ be a generic subset of $P$ (over the universe $V$ ). If we denote, for $n<\omega$,

$$
G_{n}=\left\{p^{(n)}: p \in G\right\},
$$

then formula (3.7) defines subsets $x_{n} \subset \omega_{n}$, for all $n$. A routine argument (cf. (3.4.0)) shows that $V[G]=V\left[\left\langle x_{n}: n<\omega\right\rangle\right]$.

For every $n$, let $P^{>n}$ denote the set of all $p \in P$ such that $P^{(k)}=(\varnothing, \varnothing)$ for all $k<n$, and let

$$
G^{>n}=G \cap P^{>n} .
$$

It is clear that $V\left[G^{>n}\right]$ and $V$ have exactly the same subsets of $\omega_{n}$, and therefore the same cardinals $\leqslant \aleph_{n+1}$. Also, $V\left[G^{>n}\right] \vDash 2^{\aleph_{k}}=\aleph_{k+1}$ for all $k<n$.

In $V\left[G^{>n}\right]$, consider the set of forcing conditions $P_{A}^{(n)}$ (see (3.5)), where 
$A=x_{n+1}$. It follows from condition (3.11)(iii) that

$$
\begin{gathered}
G_{n} \text { is a } V\left[G^{>n}\right] \text {-generic subset of } P_{x_{n+1}}^{(n)}, \\
V\left[G^{>n-1}\right]=V\left[G^{>n}\right]\left[G_{n}\right],
\end{gathered}
$$

and

$$
P^{>n-1} \simeq P^{>n} * P_{x_{n+1}}^{(n)} .
$$

(Here $P * Q$ denotes the iterated forcing: first $P$ and then $Q$.) From (3.8) and (3.14) we get

$$
x_{n+1} \in V\left[x_{n}\right],
$$

and, by iterating (3.15) and (3.16), we have

$$
V[G]=V\left[G^{>n}\right]\left[G_{n}\right]\left[G_{n-1}\right] \cdots\left[G_{0}\right]
$$

and

$$
P \simeq P^{>n} * P_{x_{n+1}}^{(n)} * P_{x_{n}}^{(n-1)} * \cdots * P_{x_{1}}^{(0)} .
$$

By iteration of (3.17) we obtain

$$
V[G]=V\left[x_{0}\right] .
$$

Let $B=$ r.o. $P$, and let $\mathrm{x}_{0}$ be the canonical name for $x_{0}$ in $V^{B}$. Since $x_{0} \subset \omega$, equation (3.20) proves that $V^{B}$ satisfies that the universe is obtained from $V$ by adjoining a subset $\mathrm{x}_{0}$ of $\omega$. Let $X=\left\{\llbracket n \in \mathrm{x}_{0} \rrbracket: n \in \omega\right\}$, and let $B_{X}$ be the complete subalgebra of $B$ generated by $X . B_{X}$ is countably generated and $V\left[x_{0}\right]=V\left[G \cap B_{X}\right]$ for any generic ultrafilter on $B$ (see, e.g., [3, Lemma 69]). Thus by (3.20), $V[G]=V\left[G \cap B_{X}\right]$ for all $G$. Using a lemma of Vopěnka (see [7, p. 232]), it follows that the atomless part of $B$ is isomorphic to the atomless part of $B_{X}$. Since $B$ is atomless, it is itself isomorphic to a countably generated algebra and therefore $B$ is countably generated.

To show that $B$ preserves cardinals we argue as follows: First we show that each $\aleph_{n}$ is preserved: We use (3.19). Forcing via $P$ can be viewed as iterated forcing, and each step in the iteration (3.19) preserves $\kappa_{n}$.

Therefore all $\kappa_{n}$ 's are preserved, and so is $\kappa_{\omega}$. Also, $\kappa_{\omega+1}$ is preserved: If it were not, then it would have in $V[G]$ the cofinality of some $\kappa_{n}$. Using (3.19) again we see that $\kappa_{\omega+1}$ has cofinality $>\kappa_{n}$ in $V\left[G^{>n}\right.$, and the remaining steps in the iteration (3.19) preserve cofinality $>\kappa_{n}$ (the chain condition). Finally, since $|P|=\kappa_{\omega}^{\kappa_{0}}=\kappa_{\omega+1}$, all cardinals $\geqslant \kappa_{\omega+2}$ are preserved.

It remains to show that $|B|=\kappa_{\omega+2}$. Since $|P|=\kappa_{\omega+1}$, we have $|B| \leqslant 2^{\kappa_{\omega+1}}$ $=\kappa_{\omega+2}$. To show that $|B| \geqslant \kappa_{\omega+2}$, it suffices to find a pairwise incompatible $W \subset P$ of size $\kappa_{\omega+1}$. For every $n$, take $\kappa_{n}$ pairwise incompatible 0 -1-functions $f^{(n)}$ whose domains are subsets of $\omega_{n}$ of size $<\kappa_{n}$ and let $W$ be the set of all possible $p=\left\langle p^{(n)}: n<\omega\right\rangle$ where $p^{(n)}=\left(f^{(n)}, \varnothing\right)$. Clearly, $W$ is pairwise incompatible and $|W|=\kappa_{\omega+1}$.

Thus $B$ has properties (a), (b), (c).

PosTSCRIPT. Lev Bukovsky informs me that he has obtained a similar result, using a slightly different construction. He moreover observes that the algebra is totally nonhomogeneous. 


\section{REFERENCES}

1. H. Gaifman, Infinite Boolean polynomials. I, Fund Math. 54 (1964), 229-250; errata, ibid. 57 (1965), 117. MR $29 \# 5765 ; 31$ \#2179.

2. A. W. Hales, On the non-existence of free complete Boolean algebras, Fund. Math. 54 (1964), 45-66. MR 29 \#1162.

3. T. J. Jech, Lectures in set theory, with particular emphasis on the method of forcing, Lecture Notes in Math., vol. 217, Springer-Verlag, Berlin and New York, 1971. MR 48 \# 105.

4. R. B. Jensen and R. M. Solovay, Some applications of almost disjoint sets, Mathematical Logic and Foundations of Set Theory (Proc. Internat. Colloq., Jerusalem, 1968), North-Holland, Amsterdam, 1970, pp. 84-104. MR \#6482.

5. S. A. Kripke, An extension of a theorem of Gaifman-Hales-Solovay, Fund. Math. 61 (1967), 29-32. MR 36 \#3693.

6. R. M Solovay, New proof of a theorem of Gaifman and Hales, Bull. Amer. Math. Soc. 72 (1966), 282-284. MR 32 \# 4057.

7. P. Vopénka and P. Hájek, Theory of semisets, North-Holland, Amsterdam, 1972.

Department of Mathematics, Pennsylvania State University, University Park, PennSYLVANIA 16802 